\title{
Prevalencia de Mammomonogamus laryngeus (Strongylida: Syngamidae) en ganado bovino de la central de beneficio del municipio de La Tebaida, Quindío, Colombia
}

\author{
Diana Marcela Echeverry ${ }^{1,2}$, Juan Miguel Rengifo', Jhon Carlos Castaño², Germán Alberto Téllez², \\ María Mercedes González ${ }^{2}$ \\ 1 Programa de Biología, Facultad de Ciencias Básicas y Tecnológicas, Universidad del Quindío, Armenia, \\ Colombia \\ 2 Grupo de Inmunología Molecular, Centro de Investigaciones Biomédicas, Facultad de Medicina, Universidad del \\ Quindío, Armenia, Colombia
}

Introducción. Mammomonogamus laryngeus es un parásito nematodo que suele encontrarse en la laringe de mamíferos del trópico, especialmente en bovinos, ovejas y gatos.

Objetivo. Determinar la prevalencia de M. laryngeus en bovinos de la central de beneficios del municipio de La Tebaida, Quindío, Colombia.

Materiales y métodos. Para determinar la presencia de infestación por M. laryngeus, se examinó la tráquea y el árbol pulmonar de los animales bovinos sacrificados en la central de sacrificio de La Tebaida, entre diciembre de 2008 y febrero de 2009.

Resultados. Se examinaron 500 bovinos. La prevalencia de infestación por M. laryngeus fue de 14,8 $\%$ (74); las formas adultas del parásito se localizaron con mayor frecuencia en el repliegue aritenoepiglótico y en ningún caso en la tráquea o bronquios. Los departamentos del país de donde provenían los animales con mayor prevalencia, fueron: Valle del Cauca (10\%), Quindío (3,6 \%) y Caquetá $(1,2 \%)$.

Conclusiones. El reporte de esta parasitosis en animales bovinos procedentes de otros departamentos, indica la circulación del parásito en Colombia. Por ende, se hace necesario hacer un estudio para definir la prevalencia actual de esta parasitosis en ganado para consumo humano.

Palabras clave: infecciones por Strongylida, enfermedades de los bovinos, interacciones huéspedparásitos, prevalencia, epidemiología, Colombia.

Prevalence of Mammomonogamus laryngeus (Strongylida: Syngamidae) nematodes in a bovine slaughterhouse

Introduction. Mammomonogamus laryngeus is a nematode parasite that is usually found in the larynx of mammals in the tropics, especially in cattle, sheep and cats.

Objective. The prevalence of $M$. laryngeus was determined in cattle brought to an abattoir as an indicator of its prevalence in the general population.

Materials and methods. The present study was undertaken in the municipality of La Tebaida, Quindio, Colombia, to determine the presence of $M$. laryngeus infection in cattle brought to a local abattoir between December and February 2009. The tracheae and lungs of 500 slaughtered cattle were examined.

Results. Parasite prevalence was $14.8 \%(n=74)$. The majority of worms were located on arytenoepiglottic folds, but never in the tracheae or bronchi. The infection rate was highest (47\%) in 2 to 2.5 year old animals. In infected animals, an average of 6.4 parasite pairs was found, with higher numbers in older animals. The prevalence of infection in cattle was greater than seen in the provinces of Valle del Cauca (10.0\%), Quindío(3,0\%) pr Caquetá (1.2\%).

Conclusions. Reports of this parasite in cattle indicate its circulation throughout Colombia and recommend surveillance studies where cattle are raised and prepared for human consumption.

Key words: Strongylida infections, cattle diseases, host-parasite interactions, prevalence, epidemiology, Colombia.

\section{Participación de los autores:}

Diana Marcela Echeverry y Juan Miguel Rengifo: toma y procesamiento de muestras; participación en la elaboración de la primera versión del manuscrito.

Jhon Carlos Castaño: revisión de datos y elaboración del manuscrito.

Germán Alberto Téllez: asesoría técnica de laboratorio.

María Mercedes González: asesoría en el análisis de datos. 
Mammomonogamus (Syngamus) laryngeus (del griego mammo, mama; mono, único; gamos, matrimonio) (1), pertenece a la familia Syngamidae. Es un parásito hematófago que se localiza en tráquea y laringe de animales bovinos, bubalinos y, ocasionalmente, de ovinos, caprinos y cérvidos $(2,3)$. Sólo el género Mammomonogamus parasita a los humanos (3).

La hembra y el macho se encuentran permanentemente en cópula y asumen la forma característica de "Y" mayúscula, donde la hembra corresponde al brazo largo de la letra y el macho al corto (figura 1). Los huevos depositados por el parásito en el moco de la tráquea son deglutidos y eliminados en las materias fecales. En el medio exterior, las larvas infecciosas (de tercer estadio) pueden desarrollarse dentro del huevo o fuera de él.

Los huevos no tienen opérculo, tienen una forma elipsoide u ovoide y miden alrededor de $45 \times 80$ $\mu \mathrm{m}$ (4) (figura 2). La mayoría de las veces, la mamomonogamiasis se diagnostica cuando el animal parasitado es sacrificado. En el hombre, el diagnóstico suele efectuarse por la integración de la sintomatología clínica y la broncoscopia.

El ciclo de vida del parásito no está bien dilucidado aún; sin embargo, se piensa que puede ser similar al que se ha descrito en las aves infectadas con Syngamus trachea $(5,6)$. Los reservorios de $M$. laryngeus son los rumiantes. El hombre se infecta sólo de modo accidental. Para los rumiantes, las fuentes de infestación son el suelo, el pasto y el agua.

Las manifestaciones clínicas de $M$. laryngeus se producen como consecuencia de la invasión del sistema respiratorio, principalmente de la mucosa de las vías respiratorias superiores, y consiste fundamentalmente en tos crónica, que puede ser con expectoración o, más frecuentemente, no productiva, síntomas asmatiformes y molestia laríngea. En algunos pacientes, la expectoración es hemoptoica $(7,8)$.

La infestación en humanos es poco frecuente. Actualmente, se han reportado poco más de 100 casos por $M$. laryngeus, la mayoría en pacientes que visitaron Brasil o las islas del Caribe, de los

\section{Correspondencia:}

Jhon Carlos Castaño, Facultad de Medicina, Universidad del Quindío, Carrera 15 calle 12 norte, Armenia, Colombia

Telefax: (+57) 67460129

jhoncarlos@uniquindio.edu.co

Recibido: 21/01/11; aceptado:12/04/11

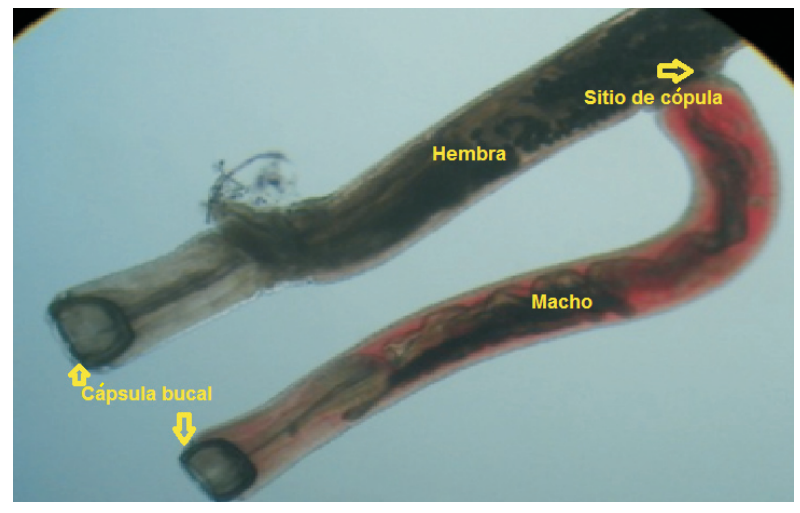

Figura 1. Identificación de la hembra y el macho en permanente cópula, con su cápsula bucal en forma de copa. Microscopio invertido Olympus CK2®, 10X.

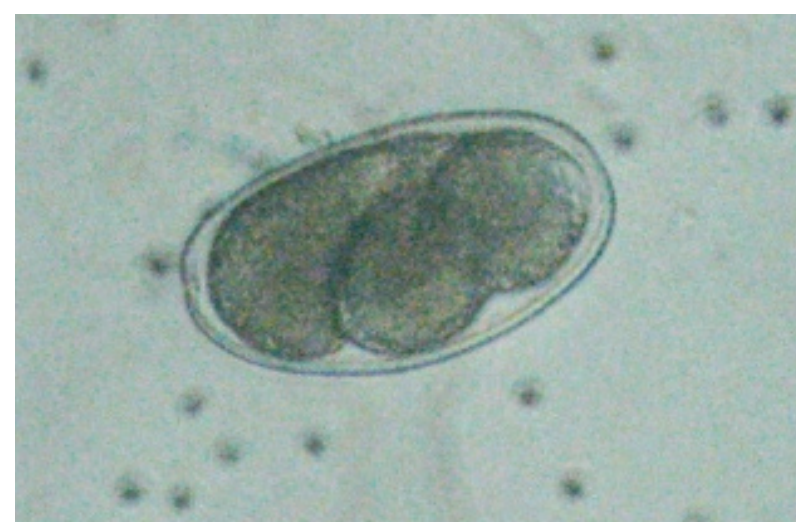

Figura 2. Huevos de Mammomonogamus laryngeus con un tamaño de $45 \times 80 \mu \mathrm{m}$. Se puede apreciar la ausencia de opérculo. Microscopio invertido Olympus $\mathrm{CK} 2 \AA, 40 \mathrm{X}$.

cuales, aproximadamente, la mitad se originó en la isla de Martinica (9). En Norteamérica se han reportado cinco casos, y en todos las personas refirieron haber visitado la zona del Caribe $(4,10,11)$. La singamosis humana se ha reportado en las islas del Caribe y en Suramérica, especialmente en Brasil; otros reportes provienen de Australia (6), Canadá (8), Estados Unidos $(4,8,12)$, Reino Unido $(13,14)$ y Francia $(15,16)$. En el continente asiático también existen casos reportados en Filipinas, Corea y Tailandia, aunque se piensa que en algunos de ellos las infestaciones habían sido adquiridas en China, Vietnam y Malasia (3).

En Colombia hasta ahora sólo se ha reportado un caso clínico de esta parasitosis en humanos, en quien los únicos antecedentes epidemiológicos habían sido el contacto con gatos domésticos y los viajes recreativos frecuentes a fincas de la región donde existe ganado bovino, situación que podría representar la posible presencia de esta parasitosis en la región (17). 
A nivel mundial se han reportado tasas de prevalencias por $M$. laryngeus en animales bovinos en países como Brasil; en un matadero del estado de São Paulo se encontraron infectadas 27 (45\%) de 60 vacas sacrificadas (18) y en el estado de Río de Janeiro reportaron 18 infectados $(37,5 \%)$ de 48 novillos (19). En Honduras, se encontraron parásitos sólo en 2,8\% de 70 bovinos examinados. En Venezuela, se reportó que $14,2 \%$ de 618 animales bovinos estaban parasitados (20). En Filipinas, $23 \%$ de 597 animales vacunos estaban parasitados con $M$. laryngeus $(21,22)$. En Colombia sólo se ha realizado un reporte de esta parasitosis en animales bovinos en 1977 en el departamento del Valle del Cauca (23). Asimismo, el primer caso de esta entidad en humanos se reportó en el departamento del Quindío, lo cual sugiere la presencia del parasito en el ambiente.

El objetivo de este trabajo fue determinar la prevalencia de $M$. laryngeus en ganado bovino sacrificado en la central de beneficio del municipio de La Tebaida, Quindío.

\section{Materiales y métodos}

Se hizo un estudio de corte transversal, en los animales bovinos sacrificados en la central de beneficio del municipio de La Tebaida, Quindío, durante dos meses, entre el 13 de diciembre de 2008 y el 10 de febrero de 2009, hasta completar 500 animales.

En cada uno de los animales se observó en forma directa la laringe, la tráquea y los bronquios para determinar la presencia del parásito. Se recolectó información epidemiológica sobre variables como: lugar de procedencia, fecha de toma de la muestra, sexo, edad y peso. Los cálculos estadísticos se hicieron con el programa Stagraphics Centurion $X V \AA$. Para determinar la prevalencia, se utilizó la frecuencia porcentual simple, y luego se relacionaron las variables frente a los resultados obtenidos, utilizando un modelo de regresión logística.

\section{Consideraciones éticas}

Para el presente estudio se contó con la autorización de la gerencia de la central de sacrificio del municipio de La Tebaida. Las muestras se tomaron en los animales después del sacrificio, el cual se llevó a cabo según las directrices de la Food and Agriculture Organization (FAO) para el manejo, transporte y sacrificio humanitario del ganado (24).

\section{Resultados}

De 500 animales bovinos estudiados, se encontraron 74 parasitados por larvas adultas de $M$. laryngeus (14,8\%), siendo la proporción de machos ligeramente mayor $(20,4 \%)$ en relación con las hembras $(10,4 \%)$ (cuadro 1).

En las observaciones macroscópicas, se evidenció que el sitio de predilección de las formas adultas era el repliegue ariteno-epiglótico (82,4\%) (figura 3) y que el número de especímenes por bovino varió de 1 a 62 parejas, y en todos los casos se observó al macho unido a la hembra. En el examen practicado no se pudieron identificar parásitos localizados en la tráquea ni en los bronquios principales o secundarios.

Los animales sacrificados eran procedentes del departamento del Quindío y departamentos aledaños, encontrándose que el de procedencia con mayor prevalencia fue Valle del Cauca (10\%), seguido por Quindío (3,6 \%) y Caquetá (1,2\%) (figura 4).

En el análisis estadístico de los datos consignados en los registros epidemiológicos, se encontró que la población estudiada presentaba un mayor porcentaje en el grupo de edad de dos años $(84,2 \%)$ para los bovinos sacrificados (cuadro 2). Igualmente, el análisis estadístico demostró que el porcentaje de animales infectados por $M$. laryngeus fue similar al de los sanos en el grupo de

Cuadro 1. Prevalencia según sexo de Mammomonogamus laryngeus en bovinos sacrificados en la central de beneficio de La Tebaida, Quindío, diciembre de 2008 a febrero de 2009.

\begin{tabular}{lccc}
\hline $\begin{array}{l}\text { Sexo de los animales } \\
\text { examinados }\end{array}$ & $\mathbf{n}$ & Positivos & $\%$ \\
\hline Hembras & 279 & 29 & 10,39 \\
Machos & 221 & 45 & 20,36 \\
Total & 500 & 74 & 14,80 \\
\hline
\end{tabular}

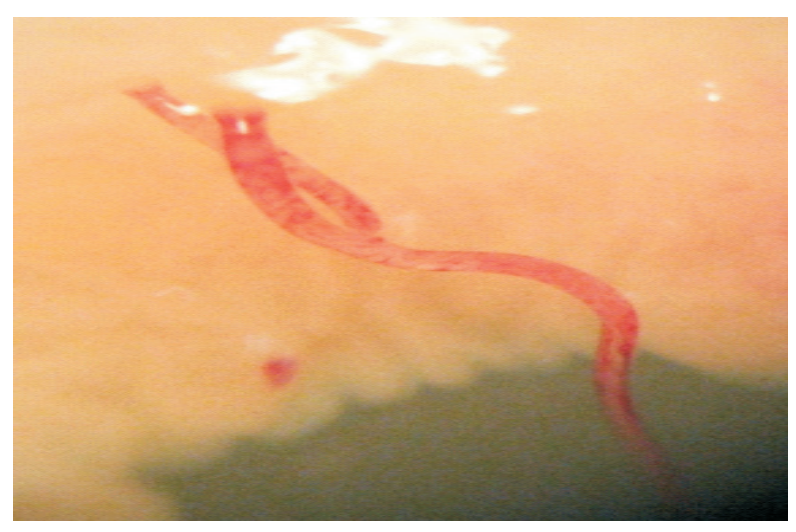

Figura 3. Mammomonogamus laryngeus en el repliegue aritenoepiglótico de uno de los bovinos de la central de beneficio del municipio de La Tebaida, Quindío; nótese su característica forma en Y. Estereoscopio. 


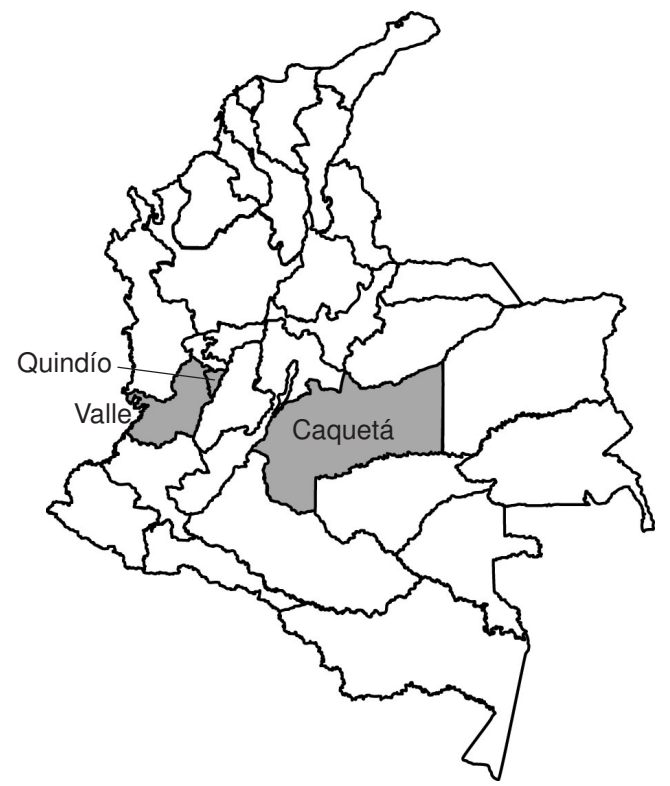

Cuadro 2. Porcentaje de bovinos (Bos taurus) infectados con Mammomonogamus laryngeus, según los grupos de edad, en la central de beneficio de La Tebaida, Quindío, diciembre de 2008 a febrero de 2009.

\begin{tabular}{lrcc}
\hline & & \multicolumn{2}{c}{ Infestación por } \\
& & Mammonogamus laryngeus \\
\cline { 3 - 4 } Grupo de edad (años) & Total & $\begin{array}{c}\text { Presente } \\
\mathbf{n}(\%)\end{array}$ & $\begin{array}{c}\text { Ausente } \\
\mathbf{n}(\%)\end{array}$ \\
& & $23(54,8)$ & $19(45,2)$ \\
$\leq 1$ cumplido & 42 & $50(11,9)$ & $371(88,1)$ \\
2 cumplidos & 421 & $1(2,7)$ & $36(97,3)$ \\
3 cumplidos & 37 & & \\
\hline
\end{tabular}

reses menores de un año $(p>0,05)$. Sin embargo, tanto en el grupo de dos años cumplidos como en el de tres, la frecuencia de animales infectados fue menor que el de no infectados $(p<0,01)$. Finalmente, se encontraron diferencias en los porcentajes de infestación en los diferentes grupos de edades $(p<0,01)$, con una tendencia a disminuir a medida que aumentaba la edad $(p<0,01)$.

En el análisis de regresión logística se encontró que la edad $(p<0,001)$, el sexo $(p<0,047)$ y la procedencia del bovino $(p<0,0022)$, se asociaban con la presencia o ausencia del parásito.

Cuando se compararon los porcentajes de los bovinos parasitados con el lugar de procedencia, se halló una razón de probabilidad de 6,61 para los procedentes del municipio de Montenegro (Quindío), por lo que la probabilidad de encontrar un bovino parasitado y que fuera del Quindío, era seis veces mayor en comparación con los procedentes de otros departamentos.

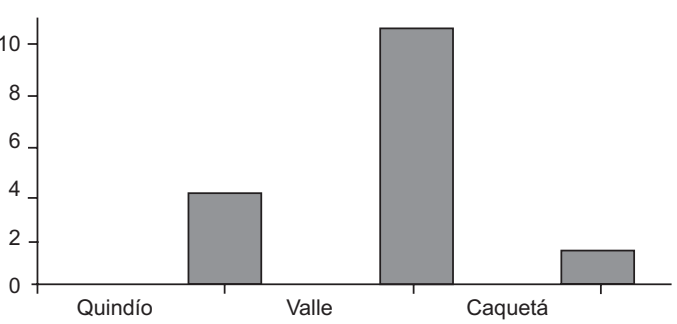

Figura 4. Distribución de Mammomonogamus laryngeus según el lugar de procedencia de los bovinos sacrificados en la central de beneficio del municipio de La Tebaida, Quindío, diciembre de 2008 a febrero de 2009. En el mapa de Colombia se han resaltado los departamentos de donde procedían los animales estudiados.

\section{Discusión}

Los estudios epidemiológicos de vigilancia de enfermedades con potencial zoonótico, como la mamomonogamosis, en las centrales de sacrificio de animales destinados al consumo humano son de gran importancia, dado que se garantiza el control de calidad del producto para el consumidor final. Igualmente, permiten conocer el riesgo potencial para los empleados de estas instituciones y establecer medidas sanitarias profilácticas para los mismos.

La frecuencia de $M$. laryngeus en la central de beneficios de La Tebaida fue de $14,8 \%$, lo que demuestra la presencia y circulación de esta parasitosis en el entorno ecológico de donde provienen estos animales. Por lo tanto, se hace necesario crear programas de vigilancia y control por parte de las entidades de salud pública del municipio, que permitan concientizar a la comunidad general, así como a aquellas personas dedicadas a la explotación ganadera, veterinarios y a los consumidores, y así evitar la propagación de esta parasitosis mediante campañas educativas.

Contrario a lo reportado por Brown (25), quien señala que $M$. laryngeus se ubica en las vías respiratorias inferiores de los rumiantes, en el presente estudio en ninguno de los animales se observaron parásitos en el árbol bronquial. Las formas adultas se localizaron principalmente en el repliegue ariteno-epiglótico y en ningún caso en la tráquea o los bronquios, resultados similares a 
los encontrados por Villegas, et al. (21), quienes observaron que el sitio de predilección de las formas adultas de este parasito era el repliegue ariteno-epiglótico.

El hallazgo de que la mayoría de los bovinos parasitados eran machos, concuerda con lo reportado por Villegas, et al., en Venezuela, quienes encontraron una proporción de machos ligeramente mayor $(15,6 \%)$ en relación con las hembras (12,0 \%) (21). La mayor incidencia en animales con un peso entre 360 y $460 \mathrm{~kg}$ se debe a que, en esta central, el mayor número de animales sacrificados en este periodo correspondió a bovinos con este peso, como se observó en los registros de acopio de los animales que ingresaron de diciembre a febrero. Esto puede deberse a que éste sea el promedio estándar que se comercializan en condiciones normales y son sacrificados para obtener la mayor utilidad económica por parte del propietario. Esto no significa que haya una relación directa que indique que los bovinos con mayor peso son más propensos a adquirir la parasitosis que los de menor peso, ya que en todos los pesos registrados se evidenciaron positivos.

Los estudios epidemiológicos son de vital importancia para la detección de focos de infestación, ya que localizarlos puede ser de gran ayuda puesto que se puede pensar en impartir educación a las personas que hagan parte del mismo, con el fin de mejorar sus hábitos de higiene y alimentarios, entre otros, para de esta manera, tal vez, contrarrestar un poco el alto índice de infestación que se presenta actualmente. Tanto en el campo médico como en el veterinario, los estudios epidemiológicos de prevalencia que se han reportado indican que en el departamento del Quindío existe circulación del parásito, como se pudo corroborar previamente en el primer caso humano demostrado en Colombia, el cual fue un paciente de Armenia que pasó una temporada vacacional en La Tebaida antes de que le aparecieran los síntomas (17).

La procedencia de animales del municipio de Montenegro (Quindío) se asoció con una razón de probabilidad de 6,61, lo cual muestra que en nuestro departamento se presentan las condiciones óptimas para el mantenimiento del ciclo de transmisión de este parásito. Además, si consideramos que los animales sacrificados en la central de sacrificio de La Tebaida en los que se encontró el parasito adulto procedían de departamentos como Valle del Cauca y Caquetá, se infiere la circulación de M. laryngeus en una extensa área geográfica de nuestro país. Por esta razón, se hace necesario hacer un estudio para establecer la prevalencia actual de esta parasitosis en ganado para consumo humano en Colombia, así como brindar la posibilidad de desarrollar una prueba serológica para el diagnóstico en animales y humanos con sintomatología clínica sugestiva de esta enfermedad, y así poder determinar la verdadera importancia de la mammomonogamosis en nuestro medio.

La prevalencia de $14,8 \%$ para $M$. laryngeus en bovinos sacrificados en el municipio de La Tebaida, puede considerarse intermedia en la región, pues fue superior a lo reportado en Honduras $(2,8 \%)$ y cercana a la de Venezuela $(14,2 \%)(20,21)$, pero inferior a los hallazgos en Brasil, donde oscilaron entre $37,5 \%$ y $45 \%(18,19)$.

Finalmente, se hace necesario continuar el estudio de esta parasitosis en el departamento del Quindío y en Colombia, tanto a nivel veterinario como humano, dado que en con este trabajo se pudo establecer la circulación de $M$. laryngeus en el territorio nacional, toda vez que los animales sacrificados procedían de diferentes departamentos de Colombia.

\section{Agradecimientos}

Los autores agradecen al Centro de Investigaciones Biomédicas de la Universidad del Quindío, por su apoyo para el desarrollo de este trabajo; a la Central de beneficio de La Tebaida ,por toda la colaboración prestada, y a todos sus empleados, por permitir la realización de este trabajo en sus instalaciones. A Ana María Barrero Parra, por su valiosa contribución.

\section{Conflictos de intereses}

Los autores declaramos que no existen conflictos de intereses.

\section{Financiación}

El presente trabajo se hizo con recursos propios del Grupo de Inmunología Molecular, adscrito a la Facultad de Ciencias de la Salud de la Universidad del Quindío.

\section{Referencias}

1. Kim HY, Lee SM, Joo JE, Na MJ, Ahn MH, Min DY. Human syngamosis: The first case in Korea. Thorax. 1998;53:717-8.

2. Anderson RC, Chabaud AG, Willmott S. ClH keys to the nematode parasites of vertebrates. Keys to the genera of the superfamily Strongyloidea. Farrham Royal, Bucks, England: Commonwealth Agricultural Bureau; 1980.

3. de Lara T de A, Barbosa MA, de Oliveira MR, de Godoy I, Queluz TT. Human syngamosis. Two cases of chronic 
cough caused by Mammomonogamus laryngeus. Chest. 1993;103:264-5.

4. Nosanchuk JS, Wade SE, Landolf M. Case report of and description of parasite in Mammomonogamus laryngeus (human syngamosis) infection. J Clin Microbiol. 1995;33:998-1000.

5. Severo LC, Conci LM, Camargo JJ, Andre-Alves MR, Palombini BC. Syngamosis: Two new Brazilian cases and evidence of a possible pulmonary cycle. Trans R Soc Trop Med Hyg. 1988;82:467-8.

6. Acha PN, Szyfres B. Zoonosis and communicable diseases common to man and animals. Scientific and Technical Publication № 580. Washington, D.C: Pan American Health Organization; 2003.

7. Birrell DJ, Moorhouse DE, Gardner MA, May CS. Chronic cough and haemoptysis due to a nematode, Syngamus laryngeus. Aust NZ J Med. 1978;8:168-70.

8. Gardiner $\mathbf{C H}$, Schantz PM. Mammomonogamus infection in a human. Report of a case. Am J Trop Med Hyg. 1983;32:995-7.

9. Mornex JF, Maddeleine J, De Thore J. La syngamose humaine (Mammomonogamus nasicola) cause de toux chronique en Martinique. 37 observations recentes. Nouv Presse Med. 1980;9:3628.

10. Cunnac M, Magnaval JF, Cayarci D, Leophonte P. A propos de 3 cas de syngamose humaine en Guadalupe. Rev Pneumol Clin. 1988;44:140-2.

11. Pontes PA, Gadelha ME, Gregorio LC. Pathologic quiz case 2. Laryngeal syngamosis. Arch Otolaryngol Head Neck Surg. 1993;119:570-4.

12. Weinstein L, Molavi A. Syngamus laryngeus infection (syngamosis) with chronic cough. Ann Intern Med. 1971;74:577-80.

13. Basden RD, Jackson JW, Jones El. Gapeworm infestation in man. Br J Dis Chest. 1974;68:207-9.

14. Turner P, Turner CG, Bowers KM, Gibson DI, Chiodini PL. A case of human Syngamosis. Travel Med Infect Dis. 2003;1:231-3.
15. Junod $\mathbf{C}$, Philbert $\mathbf{M}$, Sang $\mathbf{H}$. Une observation de syngamose humaine a localisation bronchique. Premier cas traité et guéri par le thiabendazole. Bull Soc Pathol Exot Filiales. 1970;63:483-8.

16. Ho-Thi-Sang, Junod C, Philbert M. Bull Soc Pathol Exot Filiales. 1970;63:488-97.

17. Castaño JC, Núñez FA, González MM, Téllez G. Reporte del primer caso humano de infección parasitaria por Mammomonogamus laryngeus en Colombia. Biomédica. 2006;26:37-41.

18. Santos IF, Fukuda RF. Ocorrencia de Syngamus laryngeus em bovinos do Municipio de Novo Horizonte. S P Científica (S Paulo). 1977;5:391-3.

19. Freire NM, Biachin I. Prevalencia de Mammomonogamus laryngeus (Railliet, 1899) em bovinos no Rio de Janeiro. Arq Esc Vet UEMG (Minas Gerais) 1979;31:23-4.

20. Moreno LG, Gómez EA. Parásitos gastrointestinales y pulmonares en bovinos del estado Bolívar. Vet Trop. 1991;16:55-68.

21. Villegas J, Linares T, Verde O. Incidencia de Syngamus laringeus en bovinos sacrificados. Agronomía Tropical. $1975 ; 25: 517-21$

22. van Aken D, Lagapa JT, Dargantes AP, Vercruysse J. Mammomonogamus laryngeus (Railliet, 1899) infections in cattle in Mindanao. Philippines. Vet Parasitol. 1996;64:32932.

23. Guzmán VH, Adams LG, Galvin TJ. Bovine laryngeus verminosis due to Mammomonogamus laryngeus in Colombia. Revista ICA. 1977;11:81-8.

24. FAO. Directrices para el manejo, transporte y sacrificio humanitario del ganado. Fecha de consulta: 11 de febrero de 2009. Disponible en: http://www.fao.org/DOCREP/005/ X6909s/x6909s09.htm.

25. Brown H. Parasitología clínica. Tercera edición. México, D.F.: Editorial Interamericana S.A; 1969. p. 176. 